\title{
Evidence of an increased neuronal activation-to-resting glucose uptake ratio in the visual cortex of migraine patients: a study comparing ${ }^{18}$ FDG-PET and visual evoked potentials
}

Marco Lisicki ${ }^{1 \dagger}$, Kevin D’Ostilio ${ }^{1 \dagger}$, Gianluca Coppola ${ }^{2}$, Felix Scholtes ${ }^{3}$, Alain Maertens de Noordhout ${ }^{1}$, Vincenzo Parisi ${ }^{2}$, Jean Schoenen ${ }^{1}$ and Delphine Magis ${ }^{1 *}$ (D)

\begin{abstract}
Background: Migraine attacks might be triggered by a disruption of cerebral homeostasis. During the interictal period migraine patients are characterized by abnormal sensory information processing, but this functional abnormality may not be sufficient to disrupt the physiological equilibrium of the cortex unless it is accompanied by additional pathological mechanisms, like a reduction in energetic reserves. The aim of this study was to compare resting cerebral glucose uptake (using positron emission tomography $\left({ }^{18}\right.$ fluorodeoxyglucose-PET)), and visual cortex activation (using visual evoked potentials (VEP)), between episodic migraine without aura patients in the interictal period and healthy volunteers.
\end{abstract}

Methods: Twenty episodic migraine without aura patients and twenty healthy volunteers were studied. ${ }^{18} \mathrm{FDG}$-PET and VEP recordings were performed on separate days. The overall glucose uptake in the visual cortex-to-VEP response ratio was calculated and compared between the groups. Additionally, PET scan comparisons adding area under the VEP curve as a covariate were performed. For case-wise analysis, eigenvalues from a specific region exhibiting significantly different FDG-PET signal in the visual cortex were extracted. Standardized glucose uptake values from this region and VEP values from each subject were then coupled and compared between the groups.

Results: The mean area under the curve of VEP was greater in migraine patients compared to healthy controls. In the same line, patients had an increased neuronal activation-to-resting glucose uptake ratio in the visual cortex. Statistical parametric mapping analysis revealed that cortical FDG-PET signal in relation to VEP area under the curve was significantly reduced in migraineurs in a cluster extending throughout the left visual cortex, from Brodmann's areas 19 and 18 to area 7. Within this region, case-wise analyses showed that a visual neuronal activation exceeding glucose uptake was present in $90 \%$ of migraine patients, but in only $15 \%$ of healthy volunteers.

(Continued on next page)

\footnotetext{
* Correspondence: dmagis@chuliege.be

${ }^{\dagger}$ Marco Lisicki and Kevin D'Ostilio contributed equally to this work.

${ }^{1}$ Headache Research Unit, University Department of Neurology CHR, CHU de

Liège, Boulevard du 12eme de Ligne 1, 4000 Liege, Belgium

Full list of author information is available at the end of the article
} 


\begin{abstract}
(Continued from previous page)
Conclusion: This study identifies an area of increased neuronal activation-to-resting glucose uptake ratio in the visual cortex of migraine patients between attacks. Such observation supports the concept that an activity-induced rupture of cerebral metabolic homeostasis may be a cornerstone of migraine pathophysiology.

This article has been selected as the winner of the 2018 Enrico Greppi Award. The Enrico Greppi Award is made to an unpublished paper dealing with clinical, epidemiological, genetic, pathophysiological or therapeutic aspects of headache. Italian Society for the Study of Headaches (SISC) sponsors this award, and the award is supported through an educational grant from Teva Neuroscience. This article did not undergo the standard peer review process for The Journal of Headache and Pain. The members of the 2018 Enrico Greppi Award Selection Committee were: Francesco Pierelli, Paolo Martelletti, Lyn Griffiths, Simona Sacco, Andreas Straube and Cenk Ayata.
\end{abstract}

Keywords: Headache, Cerebral energy metabolism, Astrocytes, Neurophysiology

\section{Background}

Between attacks, migraine patients often exhibit abnormal sensory information processing [1]. Cortical hyper-responsivity during any kind of sensory stimulus repetition, including visual, is the most common electrophysiological feature found in episodic migraine patients during the interictal state [2]. Such alteration however, although highly prevalent among migraineurs, is by itself insufficient to entirely explain migraine's pathophysiology [3]. The fact that similar cortical reactivity profiles can be found in both migraine patients and their asymptomatic first degree relatives suggests the existence of additional pathologic mechanisms in migraine sufferers that, when associated to visual hyper-responsivity, lead to the development of the disease [3].

In parallel with cortical hyper-responsivity, cerebral metabolism has been suggested to play a major role in migraine [4-6]. Hypotheses argue that reduced mitochondrial energy and ATP levels observed in the cortex of patients between attacks [7-9] might make them unable to deal with an energetically more demanding neuronal activity $[5,10]$. This imbalance would later translate into a disruption in cortical homeostasis, with subsequent activation of the trigeminovascular system. Such view is supported by the favourable clinical response to metabolic enhancers [11] and ketogenic diet [12, 13] observed in migraine patients, as well as the capability of metabolic challenges to trigger migraine attacks [6].

${ }^{18}$ Fluorodeoxyglucose-positron emission tomography -(FDG-)PET is an imaging tool used to measure glucose uptake in brain tissue. FDG-studies in migraine patients between attacks have identified regions of reduced metabolism in limbic areas belonging to the pain/salience matrix $[14,15]$. Yet, no interictal FDG-PET abnormalities affecting the visual cortex have been reported, with the possible exception of increased glucose uptake in posterior white matter [16] . Nonetheless, in available FDG-PET studies, no attempt was made to correlate cerebral metabolism with sensory processing.
In the present study we sought to determine if interictal glucose metabolism in the visual cortex is proportional to visually-induced neuronal activation. For this purpose, we assessed cerebral glucose uptake, and recorded visual evoked potentials (VEP) in healthy volunteers and migraine patients. Our main hypothesis was that the interictal responsivity of the visual cortex in migraine patients would exceed the resting glucose uptake, rendering migraineurs vulnerable to a disruption of metabolic homeostasis in times of increased neuroenergetic demands.

\section{Methods \\ Study participants}

The study involved twenty episodic migraine without aura patients (MO; mean age (SD): $31.1( \pm 12.6), 85 \%$ fem) diagnosed in accordance with The International Classification of Headache Disorders 3rd edition (Beta version) [17] and twenty healthy volunteers (HV; mean age (SD): 36.1 ( \pm 11.4$), 75 \%$ fem) who did not report having first degree relatives suffering from recurrent headaches of any type. There were no significant differences in age or gender proportions between groups. Participants were recruited among University students or their families or via our headache clinic. None of them took any medication on a daily basis (other than the contraceptive pill), and they were all were free of any systemic or neurological disease other than migraine. Patients were not under any prophylactic treatment at the time of recordings, nor had they been for at least 30 preceding days. The mean number of monthly migraine days determined by headache diary inspection (during the month of the recordings) was $4.3 \pm$ 2.5. All patients were recorded at an interval of at least $72 \mathrm{~h}$ before and after an attack. The study was approved by the Institution's ethics committee (Centre Hospitalier Régional de la Citadelle, Liège, Belgium - protocol n ${ }^{\circ} 1422$ ) and conducted following the principles of the Declaration of Helsinki. All participants gave their written informed consent. 


\section{Visual evoked potentials recordings and analysis}

Visual evoked potentials (VEP) were used as a marker of visual cortex responsiveness. Recordings were performed in the electrophysiology laboratory of the Headache Research Unit (Neurology Department, Centre Hospitalier Régional de la Citadelle, Liège, Belgium). Subjects sat on a comfortable armchair, in a quiet room with dimmed light. Needle electrodes were placed at $\mathrm{Oz}$ (active) and $\mathrm{Fz}$ (reference) of the 10-20 EEG system. With the left eye patched, participants were instructed to fixate on a red dot in the centre of a screen displaying a black and white reversing checkerboard pattern (contrast of $80 \%$, mean luminance $50 \mathrm{~cd} / \mathrm{m} 2$ ) at temporal and spatial stimulating frequencies of $1.55 \mathrm{~Hz}$ (3.1 reversals/second) and $68^{\circ}$ respectively. Six hundred epochs, each lasting $250 \mathrm{~ms}$, were uninterruptedly recorded at a sampling rate of $5.000 \mathrm{~Hz}$ using a $\mathrm{CED}^{\mathrm{mm}}$ power 1401 device (Cambridge Electronic Design Ltd., Cambridge, UK). After DC subtraction, recordings were exported to EEGLAB [18] (an open-source MATLAB (The MathWorks Inc.) toolbox for electrophysiological signal processing), where they were band-pass filtered (low pass $100 \mathrm{~Hz}$, high pass $1 \mathrm{~Hz}$ ). Artifacted epochs exceeding two standard deviations of the channel mean limit were rejected $(<7 \%$ of epochs). We extracted the area under the curve (AUC) of each single trial, and thereafter averaged these individual values. This method is deemed to be less affected by phase synchronization and thus to provide a more straightforward measure of neuronal activation [19].

\section{FDG-PET acquisition and analysis}

PET acquisitions were made in the Radiodiagnostics Department of the Centre Hospitalier Universitaire (CHU) Sart Tilman, Liège, Belgium (Prof. R. Hustinx) using a Gemini TF PET/computed tomography (CT) scanner (Philips, Eindhoven, The Netherlands). Resting cerebral metabolism was studied $30 \mathrm{~min}$ after intravenous injection of $150 \mathrm{MBq}$ FDG. Mean blood glucose level was 89.5 (range:110-77). Subjects were injected and scanned in a dark room with minimal environmental noise. They were instructed to maintain their eyes closed during the scan. Images were reconstructed using an iterative list mode time-of-flight algorithm. Corrections for attenuation, dead-time, random and scatter events were applied. PET acquisitions were analysed using Statistical Parametric Mapping version 12 (SPM12, Wellcome Trust Centre for Neuroimaging, http:// www.fil.ion.ucl.ac.uk/spm) implemented in MATLAB 7.4.0 (MathWorks Inc., Sherborn, MA, USA). Images were first manually reoriented and hen spatially normalised into a standard stereotactic space using an MNI PET template (Montreal Neurological Institute) and smoothed using an $8 \mathrm{~mm}$ full-width-half-maximum (FWHM) isotropic kernel. We performed global normalisation by applying proportional scaling. Cerebral regions identification and masks were established using the WFU PickAtlas toolbox (Wake Forest University School of Medicine, Advanced NeuroScience Imaging Research lab (ANSIR), Winston-Salem, NC, U.S.A). PET scans were compared between groups (1) without adding any covariates, and (2) using the interaction with the averaged area under the VEP as regressor. An exploratory $(p<0.001$ uncorrected) analysis followed by a more stringent comparison ( $\mathrm{p}$ family-wise error[FWE] $<0.05$ correction at a whole brain level) were performed. Only statistically significant clusters within regions of the visual cortex are reported in the results. To estimate the neuronal activation-to-overall resting glucose uptake ratio in the visual cortex, eigenvalues corresponding to Brodmann's areas 17,18 , and 19 were extracted using a volumetric mask. The ratio between these values, and the mean area under the VEP curve was then calculated. In addition, based on the statistical parametric mapping results, for case-wise analysis a specific volumetric mask was generated upon the cluster in the visual cortex exhibiting a statistically significant difference in FDG-uptake between groups when including VEP-AUC as a covariate. By using this volumetric mask, eigenvalues corresponding to this specific region were extracted for each subject.

\section{Statistical analysis}

Statistical analyses were performed in Prism version 6.00 for Windows (GraphPad Software, La Jolla, California, USA). Continuous variables were compared using the t-test. The assumption of normality was evaluated using a Shapiro-Wilk normality test. For case-wise analyses, mean area under the VEP curve (accounting for visual activity) and eigenvalues corresponding to the specific cluster of the visual cortex exhibiting significant FDG-uptake differences (accounting for metabolic activity) were Z-transformed and paired for each subject. Proportions of participants with a visual > metabolic Z-score in each group were compared using Fisher's exact test. The significance level was set at $p<0.05$ for all statistical analyses.

\section{Results}

The mean area under the curve of VEP was significantly greater in migraine patients compared to healthy controls $\left(1091 \mu \mathrm{V}^{2} \pm 164.8\right.$ vs. $\left.362.8 \mu \mathrm{V}^{2} \pm 167.7 ; p<0.01\right)$ (Fig. 1).

Four clusters of reduced metabolism where initially observed (uncorrected $p<0.001$ level) in the visual cortex of migraine patients, but they did not withstand the correction for multiple comparisons. No clusters of increased PET signal were found in the visual cortex of migraineurs. 

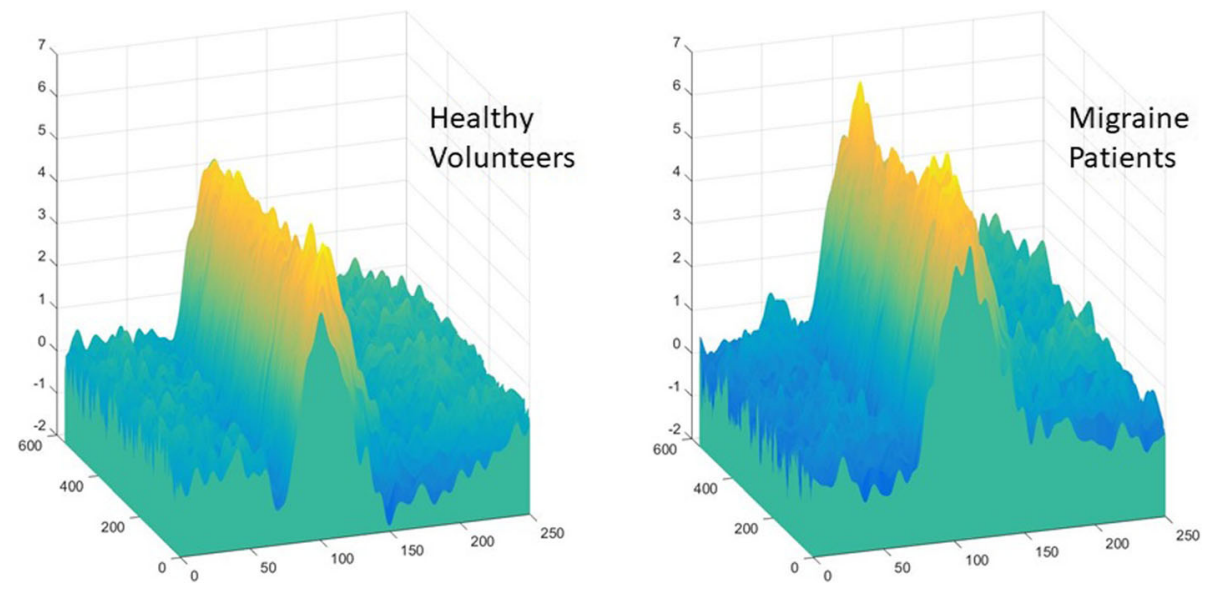

Fig. 1 Mean Visual Evoked potentials ( $x=$ time in $m s, y=$ trial number, $z=$ amplitude in $\mu \mathrm{V}$ ) in healthy volunteers (left) and episodic migraine patients (right). The area under the curve was calculated with respect to the baseline (zero)

The mean ratio between the VEP-AUC and overall glucose uptake in the visual cortex (BA 17, 18 and 19) was three times higher in migraine patients $(12,73 \pm$ 1947 vs. $4.183 \pm 1955 ; \mathrm{p}<0.01)$.

When adding VEP-AUC as a covariate, statistical parametric mapping comparisons revealed a cluster of significantly reduced uptake in patients, with three peaks extending over Brodmann's areas 19, 18 and 7 on the left $(-20,-78,30 T=5.66$ peak-level p [FWE-corr] $=0.039$; $-8,-78,20 T=4.84 ;-20,-74,42 T=4.36$, all cluster-level pFWE-corr $=0.021$ ) (Fig. 2). Eigenvalues from this specific cluster were extracted and standardized for case-wise analysis, where a visual activation Z-score exceeding the glucose uptake Z-score was found in $90 \%$ of migraine patients, but in only $15 \%$ of healthy volunteers $(p<0.001$, Fisher's exact test) (Fig. 3).

\section{Discussion}

In this study, using ${ }^{18}$ fluorodeoxyglucose-positron emission tomography acquired at rest and pattern reversal-visual evoked potentials, we compared interictal glucose uptake and neuronal activation in the visual cortex of migraine patients and healthy volunteers. Our FDG-PET data confirm the finding of an interictally decreased metabolism in the occipital areas previously shown using phosphorus magnetic resonance spectroscopy [7-9]. An additional, novel finding, is that the ratio between stimulation-induced neuronal activation and resting glucose uptake differs between migraine patients and healthy controls. Using statistical parametric mapping, we identified the specific region of the visual cortex where this difference reaches its maximum. The potential pathophysiological implications of these findings are discussed below.

According to most neurophysiological studies, migraine patients exhibit cortical hyper-responsivity to repeated sensory stimuli between attacks [20]. Although this may constitute a favourable evolutionary trait [21], enhanced responsivity has a price to be paid in terms of cerebral metabolism. In the brain, the visual system ranks amongst the most energy-consuming systems [22]. This renders it more susceptible to metabolic imbalance when energetic demands increase, energetic supplies decrease, or in a combination of both.

The occurrence of a metabolic strain in the cortex is a plausible hypothesis for igniting a migraine attack [23]. The cornerstone of the metabolic strain model is the unbalanced relationship between enhanced sensory processing and reduced metabolic offers [5]. According to this model, unmet neuronal metabolic demands in the cortex would result in a focal disruption of homeostasis and release of molecules capable of activating the trigemino-vascular system [24-26]. This mechanism, although orchestrated by neurons [26], relies on astrocytes as concertmasters [27]. By determining an area of sensory-metabolic mismatch (suggestive of the presence of a higher cortical responsivity and relatively lower resting energy reserves) in the visual cortex of migraine patients, our results provide supportive experimental evidence to the initiatory phase of the metabolic strain model. Intuitively, energy reserves may be further decreased by daily life stressful and energy consuming events [28], or by the sensory hypersensitivity that patients present even between crises, which extends up to the premonitory phase [29] when, along with the energetic deficit, may worsen up to the tipping point of the attack. This view is supported by a previous study in which the authors simultaneously recorded VEP and cerebral blood flow velocity responses (VEFR) in a group of migraineurs. They observed an interictal increase of neurovascular coupling in patients interictally that, empirically, corresponds to higher neural activity, which 


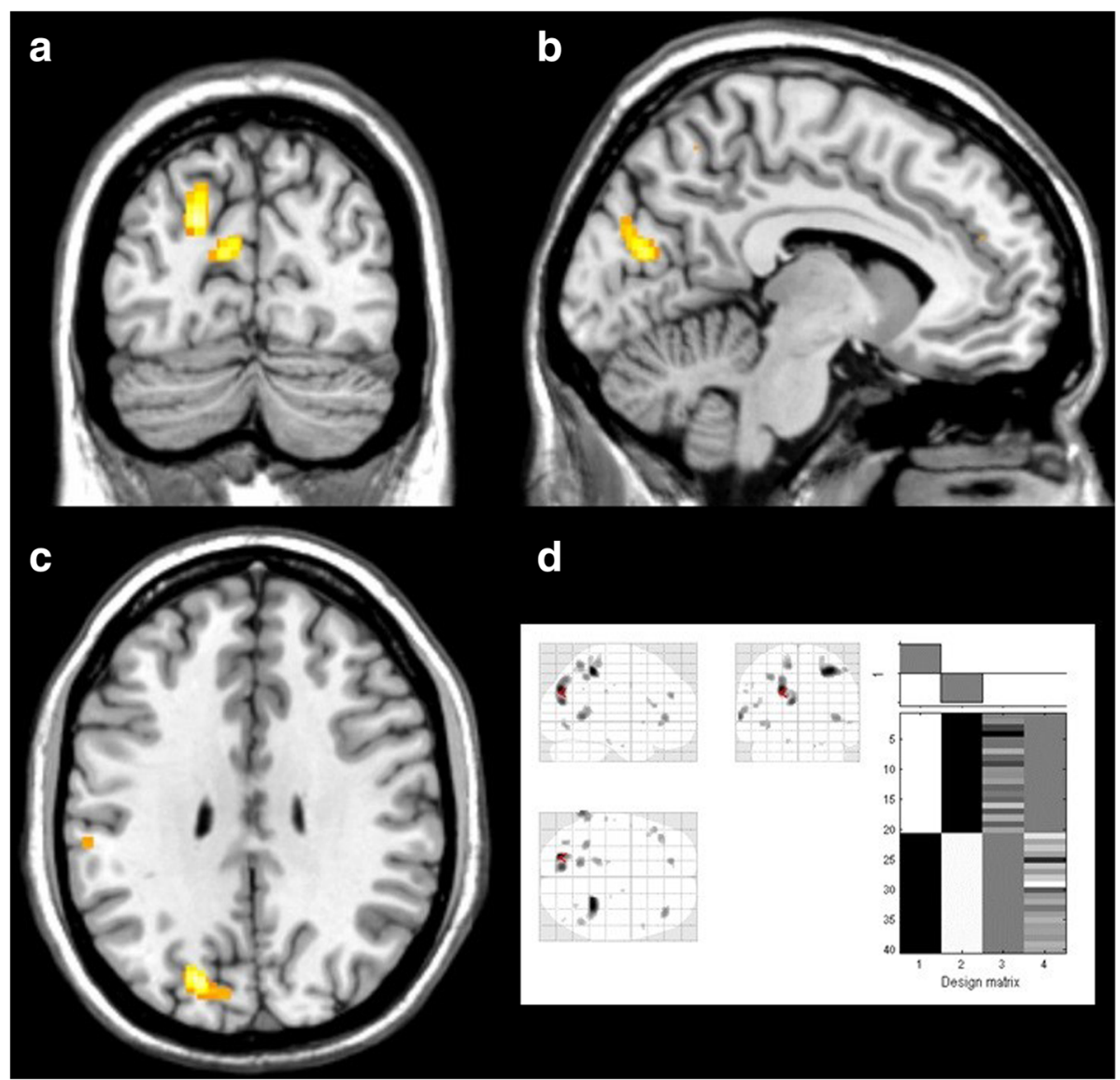

Fig. 2 Areas of increased metabolism in healthy controls compared with migraine patients using the interaction with area under the VEP as regressor. Clockwise from top left: (a) coronal view, (b) sagittal view, (c) axial view, and (d) "glass brain" representation and design matrix

might further reduce energy reserves [30]. Hence, the presence of an increase of energetic requirements in the absence of adequate energetic supplies would be a plausible reason for the brain to activate its major alarm system, the trigeminovascular system, that might ignite a migraine attack in order to prevent the system from overloading, and thus re-establish cortical homeostasis [4]. This is supported by three out of four studies where authors positively triggered migraine using sensory overload [31-34]. On the other hand, metabolic challenges have also been shown to provoke migraine attacks. From an observational perspective, skipping meals is the third most common migraine trigger reported by patients [35]. In the laboratory, Schoonman et al. reported provoking headache in six out of fourteen migraine patients exposed to normobaric hypoxia [36], and more recently, in an elegantly designed study also adressing the effects of hypoxia, Arngrim et al. reported migraine-like attacks in eight out of fifteen migraine patients, accompanied by definite aura in three, and possible aura in another four [37].
Our results do not necessarily pull in one direction, favouring sensory over metabolic factors or vice versa, but they rather imply that the physiological sensory-metabolic equilibrium is abnormal in migraine between attacks. However, it is of interest that resting brain energy metabolism remains low even during migraine attacks [38-40] when sensory hyper-responsivity tends to normalize [29]. This suggests that the former might be a trait factor permanently predisposing to the next crisis, while the latter would be more of a state-dependent phenomenon.

As mentioned before, the sensory profile of migraine patients makes them susceptible of sensory overload [1], and therefore in the need of protective mechanisms. Compensatory metaplastic changes have been hypothesized to explain the between-subject variability of electrophysiological responses in migraine groups in previous studies [41]. Thus, it could be debated whether the reduced resting glucose uptake in migraine patients we observed constitutes compensatory mechanism aiming to decrease cortical activation. However, given the heightened visual responsiveness 


\section{Z-scores}

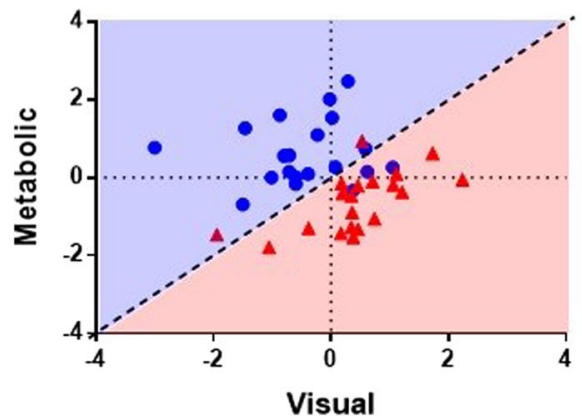

Fraction of total

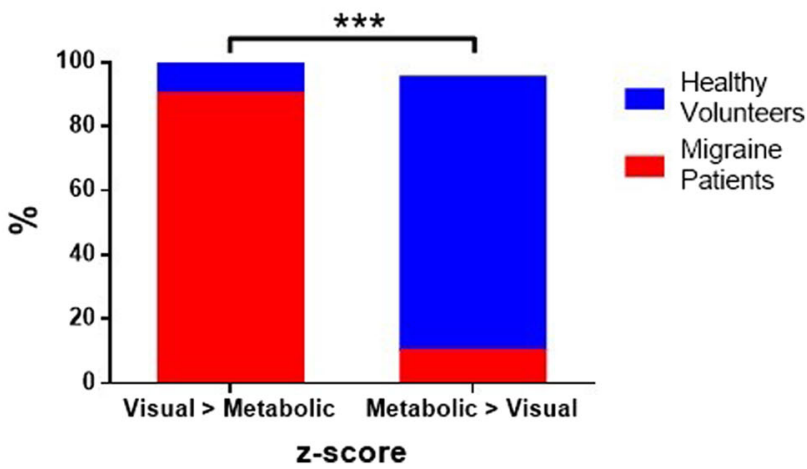

Fig. 3 Left: Metabolic and Visual Z-scores of each participant plotted on a Cartesian coordinates plane. Points above the dashed diagonal line (light blue shaded area) correspond to participants with higher glucose uptake in the visual cortex with respect to their visual responsiveness score. Points below the dashed diagonal line (light red shaded area) correspond to participants exhibiting disproportionally higher visual responses considering their glucose uptake. Right: relative proportion of subjects of each within each side of the dashed diagonal line of the Cartesian plot. The asterisks $\left(^{* *}\right)$ indicate a $p$ value $<0.001$

we recorded in patients, this alternative hypothesis seems unlikely.

One cannot discuss cortical metabolism and homeostasis without considering the role of astrocytes (a deficient uptake of glucose by astrocytes could explain our FDG-PET findings in migraine patients based on current evidence [42-44]). Neurons have a very scarce, if any, energetic reserve. Because of that, their increased metabolic demands upon stimulation basically rely on the energetic supplies provided by astrocytes. Unlike neurons, during rest astrocytes store glucose in the form of glycogen. When the cortex is stimulated, glycogen reserves in astrocytes are rapidly transformed into less complex energetic substrates, which are then provided to neurons in order to fulfil their enhanced metabolic needs (Fig. 4) [42]. In exchange, astrocytes buffer excessive glutamate molecules released in the synaptic cleft [45]. Other functions of astrocytes, besides regulating neuro-metabolic coupling and glutamate concentrations, include controlling the amount of extracellular potassium [46], and acting as regulators of cerebral blood flow [47]. Indeed, the influence of astrocytes on synaptic transmission is such, that the concept of a tripartite synapse (presynaptic neuron, astrocyte, and postsynaptic neuron) is steadily solidifying [48]. In fact, when altered, deficient clearance of potassium and glutamate by astrocytes seems to explain the pathogenesis of specific forms of migraine with monogenetic inheritance [49]. For the case of more common types of migraine, the pathophysiological implication of astrocytes is less well elucidated, although genetic studies seem to also point in their direction $[50,51]$.
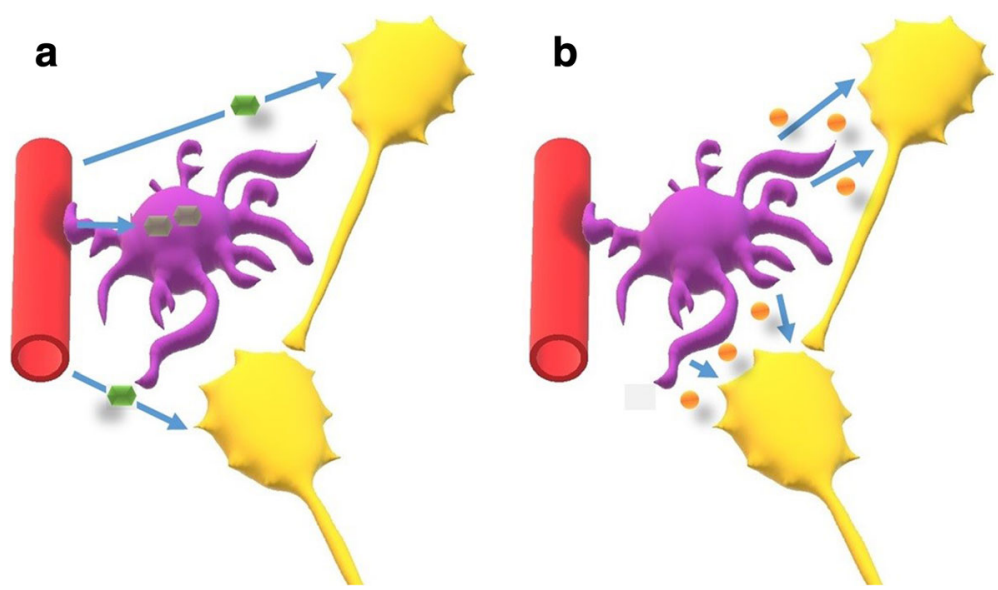

Fig. 4 Schematic representation of astrocytic (purple) and neuronal (yellow) metabolism at rest (a, left) and upon stimulation (b, right). Only astrocytes accumulate glucose (green) at rest, whereas upon stimulation, energetic reserves are degraded in order to provide energy substrates (lactate, orange) to neurons. Astrocytes in exchange reuptake exceeding glutamate at the synaptic cleft level (not shown). For details see ref. [42] 
Interestingly, the visual cortex has the lowest astrocyte/neuron density in the brain, which renders it more susceptible to homeostatic disturbances [52, 53]. Prior clinical [54] and experimental [55] evidence has suggested that waves of cortical spreading depression, the culprit of migraine aura, originate in the visual cortex. Visual area V3A was specifically pointed to as the initiating region in one report [53]. Another study described increased cortical thickness of this area in migraine without aura patients [56]. It might be worthwhile to mention that the cluster of mismatch we found in the present study extends over visual areas V3A and V7. Whether this observation is coincidental or entails clinical significance remains to be determined.

Our study has several limitations. First, PET scans and VEP where not recorded concomitantly and thus, the possibility of an important compensatory metabolic increase in migraine patients during stimulation remains open. Also, it is worth mentioning that a cycling pattern of increased susceptibility probably determined by the hypothalamus, or its limbic controls, is probably involved in migraine pathogenesis [57], and thus sensory-metabolic coupling might be preserved at one time point and impaired at another. Finally, even if there exists a metabolic imbalance during stimulation in the visual cortex of migraine patients, secondary activation of the trigeminovascular system would need to be further ascertained. In the future, studies addressing these issues would be necessary to clarify the possible role of a disruption in cortical homeostasis due to a sensory-metabolic disequilibrium in migraine pathophysiology.

\section{Conclusion}

Our findings indicate the presence of an area of increased neuronal activation-to-resting glucose uptake ratio in the visual cortex of migraine patients between attacks. Despite some methodological reservation, this observation supports the concept that an activity-induced rupture of cerebral metabolic homeostasis may be a cornerstone in migraine pathophysiology. The potential physiopathological implications of such finding should be explored in depth in future studies.

\footnotetext{
Abbreviations

${ }^{18}$ FDG-PET: ${ }^{18}$ fluorodeoxyglucose-positron emission tomography; AUC: Area under the curve; VEP: Visual evoked potentials
}

\section{Acknowledgements}

This article has been selected as the winner of the 2018 Enrico Greppi Award. The Enrico Greppi Award is made to an unpublished paper dealing with clinical, epidemiological, genetic, pathophysiological or therapeutic aspects of headache. The Italian Society for the Study of Headaches (SISC) sponsors this award, and the award is supported through an educational grant from Teva Neuroscience. This article did not undergo the standard peer review process for The Journal of Headache and Pain. The members of the 2018 Enrico Greppi Award Selection Committee were: Francesco Pierelli, Paolo Martelletti, Lyn Griffiths, Simona Sacco, Andreas Straube and Cenk Ayata.
The authors thankfully acknowledge Prof. Roland Hustinx, Chief of the Nuclear Medicine Service at the Centre Hospitalier Universitaire de Liège, for his collaboration on this study (access to FDG-PET).

The contribution of the G.B. Bietti Foundation in this paper was supported by the Italian Ministry of Health and Fondazione Roma.

\section{Funding}

This project forms part of the EUROHEADPAIN project - FP7 n 602633, and received support from the Fonds d'Investissements de Recherche Scientifique (FIRS) of the CHU de Liège.

\section{Availability of data and materials}

Further data from the underlying research material can be obtained upon request to the corresponding author.

\section{Authors' contributions}

$\mathrm{ML}$ contributed in the study design, data acquisition, data processing (focus on electrophysiology), statistical analyses, and wrote the first draft; KDO contributed in the study design, data acquisition, data processing (focus on neuroimaging) and statistical analyses; KD recruited subjects with DM; DM wrote the initial Euroheadpain protocol with JS; GC, AM de N, JS, and DM contributed in the study design and revised the drafts of the manuscript; FS and VP revised the drafts of the manuscript. All authors read and approved the final version.

\section{Ethics approval and consent to participate}

The study was approved by the Institution's ethics committee (EC number 412, Centre Hospitalier Régional de la Citadelle, Liège, Belgium - protocol nº 1422) and conducted following the principles of the Declaration of Helsinki. All participants gave their written informed consent.

\section{Consent for publication}

Not applicable.

\section{Competing interests}

The authors declare that they have no competing interests.

\section{Publisher's Note}

Springer Nature remains neutral with regard to jurisdictional claims in published maps and institutional affiliations.

\section{Author details}

${ }^{1}$ Headache Research Unit, University Department of Neurology CHR, CHU de Liège, Boulevard du 12eme de Ligne 1, 4000 Liege, Belgium. ${ }^{2} \mathrm{G}$. B. Bietti Foundation IRCCS, Research Unit of Neurophysiology of Vision and Neuro-Ophthalmology, Rome, Italy. ${ }^{3}$ Departments of Neurosurgery \& Neuroanatomy, University of Liège, Liege, Belgium.

Received: 27 April 2018 Accepted: 21 June 2018

Published online: 05 July 2018

\section{References}

1. Goadsby PJ, Holland PR, Martins-Oliveira M et al (2017) Pathophysiology of migraine: a disorder of sensory processing. Physiol Rev 97:553-622. https:// doi.org/10.1152/physrev.00034.2015

2. Magis D, Vigano A, Sava S et al (2013) Pearls and pitfalls: electrophysiology for primary headaches. Cephalalgia 33:526-539. https://doi.org/10.1177/ 0333102413477739

3. Lisicki M, Ruiz-Romagnoli E, D'Ostilio K, et al (2017) Familial history of migraine influences habituation of visual evoked potentials. Cephalalgia 37. https://doi.org/10.1177/0333102416673207

4. Schoenen J (1996) Deficient habituation of evoked cortical potentials in migraine: a link between brain biology, behavior and trigeminovascular activation? Biomed Pharmacother 50:71-78. https://doi.org/10.1016/07533322(96)84716-0

5. Schoenen J (1994) Pathogenesis of migraine: the biobehavioural and hypoxia theories reconciled. Acta Neurol Belg 94:79-86

6. Schoenen J (2016) Hypoxia, a turning point in migraine pathogenesis? Brain 139:644-647. https://doi.org/10.1093/brain/awv402

7. Reyngoudt H, Paemeleire K, Descamps B et al (2011) 31P-MRS demonstrates a reduction in high-energy phosphates in the occipital lobe of migraine 
without aura patients. Cephalalgia 31:1243-1253. https://doi.org/10.1177/ 0333102410394675

8. Montagna P, Cortelli P, Monari L et al (1994) 31 P-magnetic resonance spectroscopy in migraine without aura. Neurology 44:666-669

9. Lodi R, Montagna P, Soriani S et al (1997) Deficit of brain and skeletal muscle bioenergetics and low brain magnesium in juvenile migraine: an in vivo 31P magnetic resonance spectroscopy Interictal study. Pediatr Res 42: 866-871. https://doi.org/10.1203/00006450-199712000-00024

10. Gantenbein AR, Sandor PS, Fritschy J et al (2013) Sensory information processing may be neuroenergetically more demanding in migraine patients. Neuroreport 24:202-205. https://doi.org/10.1097/WNR. Ob013e32835eba81

11. Schoenen J, Jacquy J, Lenaerts M (1998) Effectiveness of high-dose riboflavin in migraine prophylaxis. A randomized controlled trial. Neurology 50:466-470

12. Di Lorenzo C, Coppola G, Sirianni G et al (2015) Migraine improvement during short lasting ketogenesis: a proof-of-concept study. Eur J Neurol 22: 170-177. https://doi.org/10.1111/ene.12550

13. Strahlman RS (2006) Can ketosis help migraine sufferers? A case report. Headache 46:182. https://doi.org/10.1111/j.1526-4610.2006.00321_5.x

14. Kim JH, Kim S, Suh SI et al (2010) Interictal metabolic changes in episodic migraine: a voxel-based FDG-PET study. Cephalalgia 30:53-61. https://doi. org/10.1111/j.1468-2982.2009.01890.x

15. Magis D, D'Ostilio K, Thibaut A et al (2017) Cerebral metabolism before and after external trigeminal nerve stimulation in episodic migraine. Cephalalgia 37:881-891. https://doi.org/10.1177/0333102416656118

16. Kassab M, Bakhtar O, Wack D, Bednarczyk E (2009) Resting brain glucose uptake in headache-free migraineurs. Headache 49:90-97. https://doi.org/10. $1111 / j .1526-4610.2008 .01206 . x$

17. Headache Classification Committee of the International Headache Society (IHS) (2013) The international classification of headache disorders, 3rd edition (beta version). Cephalalgia 53:137-146. https://doi.org/10.1177/ 0333102413485658

18. Delorme A, Makeig S (2004) EEGLAB: an open source toolbox for analysis of single-trial EEG dynamics including independent component analysis. J Neurosci Methods 134:9-21. https://doi.org/10.1016/j.jneumeth.2003.10.009

19. Makeig S, Debener S, Onton J, Delorme A (2004) Mining event-related brain dynamics. Trends Cogn Sci 8:204-210. https://doi.org/10.1016/j.tics.2004.03.008

20. de Tommaso M, Ambrosini A, Brighina F et al (2014) Altered processing of sensory stimuli in patients with migraine. Nat Rev Neurol 10:144-155. https://doi.org/10.1038/nrneurol.2014.14

21. Loder $E$ (2002) What is the evolutionary advantage of migraine? Cephalalgia 22:624-632

22. Wong-Riley M (2010) Energy metabolism of the visual system. Eye Brain:99. https://doi.org/10.2147/EB.S9078

23. Paemeleire K, Schoenen J (2013) (31) P-MRS in migraine: fallen through the cracks. Headache 53:676-678. https://doi.org/10.1111/head.12049

24. Zhang X, Levy D, Kainz V et al (2011) Activation of central trigeminovascular neurons by cortical spreading depression. Ann Neurol 69:855-865. https:// doi.org/10.1002/ana.22329

25. Kilic K, Karatas H, Dönmez-Demir B et al (2018) Inadequate brain glycogen or sleep increases spreading depression susceptibility. Ann Neurol 83:61-73. https://doi.org/10.1002/ana.25122

26. Karatas H, Erdener SE, Gursoy-Ozdemir Y et al (2013) Spreading depression triggers headache by activating neuronal Panx1 channels. Science 339: 1092-1095. https://doi.org/10.1126/science.1231897

27. Pietrobon D, Moskowitz MA (2014) Chaos and commotion in the wake of cortical spreading depression and spreading depolarizations. Nat Rev Neurosci 15:379-393. https://doi.org/10.1038/nrn3770

28. Lisicki M, Ruiz-Romagnoli E, Piedrabuena R, et al (2017) Migraine triggers and habituation of visual evoked potentials. Cephalalgia 333102417720217. https://doi.org/10.1177/0333102417720217

29. Judit A, Sándor PS, Schoenen J (2000) Habituation of visual and intensity dependence of auditory evoked cortical potentials tends to normalize just before and during the migraine attack. Cephalalgia 20:714-719

30. Zaletel M, Strucl M, Bajrovic FF, Pogacnik T (2005) Coupling between visual evoked cerebral blood flow velocity responses and visual evoked potentials in migraneurs. Cephalalgia 25:567-574. https://doi.org/10.1111/j.1468-2982. 2005.00918.x

31. Hougaard A, Amin F, Hauge AW et al (2013) Provocation of migraine with aura using natural trigger factors. Neurology 80:428-431. https://doi.org/10. 1212/WNL.0b013e31827fof10
32. Martin PR, Seneviratne HM (1997) Effects of food deprivation and a stressor on head pain. Health Psychol 16:310-318. https://doi.org/10. 1037/0278-6133.16.4.310

33. Cao Y, Aurora SK, Nagesh $V$ et al (2002) Functional MRI-BOLD of brainstem structures during visually triggered migraine. Neurology 59:72-78

34. Cao Y, Welch KM, Aurora S, Vikingstad EM (1999) Functional MRI-BOLD of visually triggered headache in patients with migraine. Arch Neurol 56:548-554

35. Kelman $L$ (2007) The triggers or precipitants of the acute migraine attack. Cephalalgia 27:394-402. https://doi.org/10.1111/j.1468-2982.2007.01303.x

36. Schoonman G, Sándor P, Agosti R et al (2006) Normobaric hypoxia and nitroglycerin as trigger factors for migraine. Cephalalgia 26:816-819. https:// doi.org/10.1111/j.1468-2982.2006.01112.x

37. Arngrim N, Schytz HW, Britze J et al (2016) Migraine induced by hypoxia: an MRI spectroscopy and angiography study. Brain 139:723-737. https://doi. org/10.1093/brain/awv359

38. Welch KM, Levine SR, D'Andrea $\mathrm{G}$ et al (1989) Preliminary observations on brain energy metabolism in migraine studied by in vivo phosphorus 31 NMR spectroscopy. Neurology 39:538-541

39. Welch KM, Levine SR, D'Andrea G, Helpern JA (1988) Brain pH in migraine: an in vivo phosphorus-31 magnetic resonance spectroscopy study. Cephalalgia 8:273-277. https://doi.org/10.1046/j.1468-2982.1988. 0804273.x

40. Ramadan NM, Halvorson $\mathrm{H}$, Vande-Linde A et al (1989) Low brain magnesium in migraine. Headache 29:590-593

41. Cosentino G, Fierro B, Vigneri S et al (2014) Cyclical changes of cortical excitability and metaplasticity in migraine: evidence from a repetitive transcranial magnetic stimulation study. Pain 155:1070-1078. https://doi.org/ 10.1016/j.pain.2014.02.024

42. Bélanger M, Allaman I, Magistretti PJ (2011) Brain energy metabolism: focus on astrocyte-neuron metabolic cooperation. Cell Metab 14:724-738

43. Zimmer ER, Parent MJ, Souza DG et al (2017) [18F]FDG PET signal is driven by astroglial glutamate transport. Nat Neurosci 20:393-395. https://doi.org/ 10.1038/nn.4492

44. Figley CR, Stroman PW (2011) The role(s) of astrocytes and astrocyte activity in neurometabolism, neurovascular coupling, and the production of functional neuroimaging signals. Eur J Neurosci 33:577-588. https://doi.org/ 10.1111/j.1460-9568.2010.07584.x

45. Anderson CM, Swanson RA (2000) Astrocyte glutamate transport: review of properties, regulation, and physiological functions. Glia 32:1-14

46. Kimelberg HK, Nedergaard M (2010) Functions of astrocytes and their potential as therapeutic targets. Neurotherapeutics 7:338-353. https://doi. org/10.1016/j.nurt.2010.07.006

47. Gordon GRJ, Choi HB, Rungta RL et al (2008) Brain metabolism dictates the polarity of astrocyte control over arterioles. Nature 456:745-749. https://doi. org/10.1038/nature07525

48. Araque A, Parpura V, Sanzgiri RP, Haydon PG (1999) Tripartite synapses: glia, the unacknowledged partner. Trends Neurosci 22:208-215. https://doi.org/ 10.1016/S0166-2236(98)01349-6

49. Capuani C, Melone M, Tottene A, et al (2016) Defective glutamate and K+ clearance by cortical astrocytes in familial hemiplegic migraine type 2 . EMBO Mol Med. https://doi.org/10.15252/emmm.201505944

50. Eising E, De Leeuw C, Min JL, et al Involvement of astrocyte and oligodendrocyte gene sets in migraine. https://doi.org/10.1177/ 0333102415618614

51. Renthal W Localization of migraine susceptibility genes in human brain by single-cell RNA sequencing. https://doi.org/10.1177/0333102418762476

52. Lauritzen M, Dreier JP, Fabricius M et al (2011) Clinical relevance of cortical spreading depression in neurological disorders: migraine, malignant stroke, subarachnoid and intracranial hemorrhage, and traumatic brain injury. J Cereb Blood Flow Metab 31:17-35. https://doi.org/10.1038/jcbfm.2010.191

53. Hadjikhani N, Sanchez Del Rio M, Wu O et al (2001) Mechanisms of migraine aura revealed by functional MRI in human visual cortex. Proc Natl Acad Sci U S A 98:4687-4692. https://doi.org/10.1073/pnas. 071582498

54. Lashley KS (1941) Patterns of cerebral integration indicated by the scotomas of migraine. Arch Neurol Psychiatr 46:331-339. https://doi.org/10.1001/ archneurpsyc.1941.02280200137007

55. Lauritzen M, Olesen J (1984) Regional cerebral blood flow during migraine attacks by Xenon-133 inhalation and emission tomography. Brain 107(Pt 2): $447-461$ 
56. Granziera C, DaSilva AFM, Snyder J et al (2006) Anatomical alterations of the visual motion processing network in migraine with and without Aura. PLoS Med 3:e402. https://doi.org/10.1371/journal.pmed.0030402

57. Schulte LH, May A (2016) The migraine generator revisited: continuous

scanning of the migraine cycle over 30 days and three spontaneous attacks. Brain 139:1987-1993. https://doi.org/10.1093/brain/aww097

\section{Submit your manuscript to a SpringerOpen ${ }^{\bullet}$ journal and benefit from:}

- Convenient online submission

- Rigorous peer review

- Open access: articles freely available online

- High visibility within the field

- Retaining the copyright to your article

Submit your next manuscript at $\gg$ springeropen.com 